\title{
Translocation of Klebsiella from Enteral Diets: Study through Mouse Model System and Molecular Technique
}

\author{
Pereira $\mathrm{SCL}^{1 *}$ and Maria Cristina $\mathrm{DV}^{2}$ \\ ${ }^{1}$ Federal University of Minas Gerais, School of Nursing, Department of Nutrition, Belo Horizonte-Minas Gerais, Brazil \\ ${ }^{2}$ Federal University of Viçosa, Department of Microbiology, Viçosa-Minas Gerais, Brazil
}

\begin{abstract}
Objective: This study was conducted to evaluate the translocation of Klebsiella strains to organs of normal and immunocompromised mice.

Methods: An enteral diet with $6.0 \times 10^{9} \mathrm{CFU} / \mathrm{mL}$ of Klebsiella was provided to immunodepressed and healthy animals. The presence of Klebsiella in animal organs was evaluated on MacConkey-inositol-carbenicillin agar and molecular typing was assessed by means of random amplified polymorphic DNA (RAPD).

Results: No typical Klebsiella colonies were recovered from liver, spleen, heart, kidney or lung samples when mice were supplied with the uncontaminated enteral formula. However, typical colonies were recovered from liver and lung samples from immunocompromised animals, regardless of whether they had received contaminated diet. Translocation was also detected in non-immunocompromised mice fed with contaminated diet. There were higher counts of typical Klebsiella colonies in samples collected from the guts of animals that received prednisone and/or carbenicillin. However, the highest colony count was obtained when both drugs were administered together with the enteral formula contaminated with Klebsiella. Translocation was confirmed through similarity to the DNA band patterns of orally administered Klebsiella strains.
\end{abstract}

Conclusion: K. pneumoniae was translocated into the lungs and liver of test mice fed with a Klebsiella pool. The presence of Klebsiella with a different DNA profile in liver samples from mice that only received medication suggests that autochthonous intestinal microbial strains can also become translocated when the immune system is depressed or when selective decontamination is promoted through the use of corticoids and antibiotics.

\section{Keywords: Bacterial translocation; Klebsiella; Enteral diets; RAPD}

\section{Introduction}

Klebsiella is an opportunistic pathogen that causes infections in newborns, elderly people and individuals suffering from chronic cardiac and neoplastic diseases, alcoholism, diabetes or chronic obstructive lung disease [1-3]. These infections are frequently associated with hospital environments and can result in significant mortality, especially due to exposure to several sources of contamination, or intensive therapy with immunosuppressive drugs such as corticoids and antibiotics [4].

Klebsiella pneumoniae is a capsulated Gram-negative bacterium found in the normal flora of the mouth, skin and intestine, and is also the third most commonly isolated microorganism in blood cultures from sepsis patients. Because of emerging antibiotic resistance, $K$. pneumoniae infection is becoming a major health threat [1].

Klebsiella is a common contaminant of enteral formulas [5,6], but the contribution of these diets to the epidemiology of hospital-acquired infections has so far received little attention.

In a noteworthy recent study, rapid and widespread dissemination of an epidemic clone of $K$. pneumoniae was found to cause a large nosocomial outbreak through the food chain. To our knowledge, this was the first report to provide insight on how transmission of multiresistant Klebsiella can occur through food, as the vehicle in the hospital setting [7]. To evaluate the pathogenic potential of clinical and environmental isolates of Klebsiella, virulence factors like the capsule, fimbriae, serum resistance and enterotoxin production should be taken into consideration [8]. However, bacterial pathogenesis is a complex event and varies as a function of the host-parasite relationship itself. The expression of a virulent phenotype is also influenced by environmental factors and, for this reason, can be better estimated by in vivo studies
[8]. Bacterial translocation is defined as the passage of viable bacterial cells or their endotoxins from the intestinal tract into the mesenteric lymph nodes or other organs [9]. However, in critically ill patients, with various underlying diseases, this bacterial translocation may lead to infections and consequently to a further reduction in general health status. The mechanism of bacterial translocation has been widely and somewhat controversially investigated in vitro and in animal models. In human studies, several diseases have been correlated with bacterial translocation $[9,10]$.

Translocation of orally inoculated bacteria is a proposed methodology for in vivo studies on pathogen virulence, especially regarding bacteria transmitted via contaminated food. This procedure uses the microbial ability to penetrate the intestinal epithelium and cause infections in vital organs as a virulence criterion [11].

Additionally, Klebsiella has recently been correlated with chronic intestinal diseases. The results from a large number of studies and reviews support the idea that $K$. pneumoniae is the most likely triggering factor involved in the initiation and development of ankylosing spondylitis

*Corresponding author: Pereira SCL, Federal University of Minas Gerais, Schoo of Nursing, Department of Nutrition, Belo Horizonte-Minas Gerais, Brazil, Tel: +5531-3409-8028; E-mail: simoneclpereira@gmail.com

Received November 17, 2015; Accepted December 08, 2015; Published December 21, 2015

Citation: Pereira SCL, Maria Cristina DV (2015) Translocation of Klebsiella from Enteral Diets: Study through Mouse Model System and Molecular Technique. J Food Process Technol 7: 542. doi:10.4172/2157-7110.1000542

Copyright: (c) 2015 Pereira SCL, et al. This is an open-access article distributed under the terms of the Creative Commons Attribution License, which permits unrestricted use, distribution, and reproduction in any medium, provided the original author and source are credited. 
and Crohn's disease [12]. Members of the family Enterobacteriaceae, particularly the species Klebsiella oxytoca, have been found to be more abundant in patients with active celiac disease than in controls [13].

Given this scenario, the present study aimed to evaluate the potential for Klebsiella strains isolated from enteral diets to infect vital organs and colonize the gut of experimental mice, using a molecular technique.

\section{Materials and Methods}

\section{Bacterial strains}

Five K. pneumoniae strains and one K. oxytoca strain isolated from hospital enteral diets were selected based on RAPD analysis (data submitted for publication). Serotypes, mucoid appearance present by colonies and antibiotypes were also taken into consideration during the selection (Table 1). Cultures were kept frozen at lower than $-18^{\circ} \mathrm{C}$ in trypticasein-soy broth (TSB) and glycerol. They were thawed at room temperature and reactivated twice in TSB prior to use

\section{Cell preparation}

Activated cells of Klebsiella isolates were harvested by means of centrifugation at $5000 \mathrm{~g}$ for $15 \mathrm{~min}$. Cells from all six cultures were suspended in $2 \mathrm{~mL}$ of enteral formula (Ensure, Abbott) of vanilla flavor, which had previously been prepared following the manufacturer's recommendations. Aliquots of the contaminated diets were diluted and plated onto trypticasein-soy agar in order to estimate the number of viable cells per milliliter of diet. The six cell concentrates were joined in the same flask and the volume was completed to $20 \mathrm{~mL}$. The final concentration of each isolate in this mixture was approximately $10^{10}$ colony-forming units per milliliter $(\mathrm{CFU} / \mathrm{mL})$. All procedures were done under strict sterile conditions.

\section{Animals}

Five to six-week-old Swiss albino mice weighing between 22 and 26 $\mathrm{g}$ were randomized into groups of 30 animals that were housed in cages kept in temperature-controlled rooms $\left(25^{\circ} \mathrm{C}\right)$.

\section{Experimental design}

The experiments were conducted under conditions of strict observance of ethical guidelines on animal experimentation and consisted of seven treatments (Groups I to VII) and one repetition. The baseline group (Group I) consisted of six mice that were fed standard chow and water ad libitum. The control group (Group II) consisted of 30 mice orally provided with enteral formula (Ensure ${ }^{\circledR}$, Abbot) throughout the study period. The test groups (Groups III-VI), with 30 animals each, received the enteral diet inoculated with the Klebsiella pool. Mice in GIII were solely provided with this diet; those in GIV were also given prednisone $(10 \mathrm{mg} / \mathrm{kg} /$ day); those in $\mathrm{GV}$, carbenicillin $(200$ $\mathrm{mg} / \mathrm{kg} /$ day); and those in GVI, corticoid plus antibiotic. Prednisone and carbenicillin were administered to the mice in GVII in the same way as in GVI. The animals in GVII were considered to be a second control group because they did not receive the contaminated diet. Four days after corticoid and antibiotic had been administered to the mice in GIII to GVI, all the animals received $100 \mu \mathrm{L}$ of the enteral diet inoculated with the Klebsiella pool, by means of oral nutrition. Only carbenicillin $(200 \mathrm{mg} / \mathrm{kg} /$ day $)$ was continually administered until the end of the study period. One, two, four, six and nine days after oral administration of the bacterial pool, six animals in each group were sacrificed in order to detect the presence of Klebsiella in the liver, heart, spleen, kidney, lung and gut.

\section{Translocation of Klebsiella to different organs}

Immediately after the animals were killed, their spleen, heart, lung, liver, gut and kidney were collected, weighed and stored in sterile plastic bags (Whirl-Pak, Millipore). On day nine, typical Klebsiella colonies obtained from the intestinal tracts of the animals in GII-VII were also counted. The organs were macerated and dilutions were plated onto MacConkey-inositol-carbenicillin agar (Merck) [14]. Cultures were incubated at $37^{\circ} \mathrm{C}$ for $48 \mathrm{~h}$; Petri dishes containing more than 20 colonies were selected and the number of CFU of Klebsiella per gram in each organ was estimated. Three to five typical Klebsiella colonies were picked from the same organ of each group on the sampling day, for RAPD analysis.

\section{Genetic analysis on translocated Klebsiella}

RAPD was used to identify which orally administered isolates became translocated from the intestinal tract into the sampled organs. For this reason, total DNA from the six Klebsiella cultures given to the animals and from the Klebsiella isolated from the different organs was extracted [15]. The $25 \mu \mathrm{l}$ reaction mixture consisted of $10 \mathrm{mM}$ of Tris- $\mathrm{HCl}$ ( $\mathrm{pH} \mathrm{8.0),} 50 \mathrm{mM}$ of $\mathrm{KCl}, 2.0 \mathrm{mM}$ of $\mathrm{MgCl}_{2}, 0.1 \mathrm{mM}$ of dNTP, $0.4 \mu \mathrm{M}$ of RAPD primer, $1.0 \mathrm{U}$ of Taq DNA polymerase (Promega) and $25 \mathrm{ng}$ of total DNA. The primers OPD03, OPD07, OPD08, OPD12, OPD18, OPD20, OPF10 and OPF13 were purchased from Operon Technologies Inc., Alameda, CA, USA. Thermal cycling was performed in a PTC 100 machine (MJ Research Inc., Waterton, MA, USA), programmed for an initial denaturation step at $94^{\circ} \mathrm{C}$ for $5 \mathrm{~min}$, followed by 30 cycles of $1 \mathrm{~min}$ at $94^{\circ} \mathrm{C}, 1 \mathrm{~min}$ at $40^{\circ} \mathrm{C}$, and 1.30 min at $72^{\circ} \mathrm{C}$. A final extension at $72^{\circ} \mathrm{C}$ for 7 min was performed. The amplification products were analyzed by means of electrophoresis on $1.5 \%$ agarose gels in order to facilitate the electrophoresis analysis. The gels were photographed under UV light using the Eagle-Eye II video system (Stratagene, La Jolla, CA, USA).

\section{Results}

No typical Klebsiella colonies were observed when samples of liver, spleen, heart, kidney and lung collected from mice in the baseline and control groups (GI and GII) were plated onto the selective medium.

\begin{tabular}{|c|c|c|c|c|c|}
\hline $\mathbf{N}^{\mathbf{0}}$ & Species & Isolate & Capsular serotype & Aspect mucoid & Antibiotic resistance \\
\hline 1 & K. oxytoca & P4 & SND & Absent & AMO e AMP \\
\hline 2 & K. pneumoniae & P14 & SNH & Absent & AMC, NAL, AMI, AMO, AMP, ATM, CFL, CTX, KN, NO, TET e TIC \\
\hline 3 & K. pneumoniae & P15 & SNH & Weak & AMO, AMP, ATM, KN e TET \\
\hline 4 & K. pneumoniae & U4 & SNH & Intense & NAL, AMO, AMP, ATM, CFL, CTX e TET \\
\hline 5 & K. pneumoniae & U5 & K4 & Absent & AMO e AMP \\
\hline 6 & K. pneumoniae & U8 & K5 & Absent & AMC, NAL, AMO, AMP, ATM, CFL, CTX, NO e TET \\
\hline
\end{tabular}

SND: serotype not determined. SNH: serotyping nonspecific but higher than K6. Amoxicillin + Clavulanic Acid-AMC, Nalidixic acid-NAL, Amikacin-AMI, Amoxicillin-AMO, Ampicillin -AMP, Aztreonam-ATM, Cephalothin-CFL, Cefotaxime CTX, Neomycin-NO, Tetracycline-TET, Ticarcillin + clavulanic acid-TIC.

Table 1: Klebsiella strains isolated from enteral formulas and employed for translocation studies. 
Citation: Pereira SCL, Maria Cristina DV (2015) Translocation of Klebsiella from Enteral Diets: Study through Mouse Model System and Molecular Technique. J Food Process Technol 7: 542. doi:10.4172/2157-7110.1000542

Page 3 of 5

However, they were detected in liver and lung samples from animals in GIII to GVII (Figure 1). Klebsiella could also be detected five and six days after administration of prednisone and carbenicillin, in liver samples from animals that had not been fed with a contaminated diet (GVII). RAPD analysis did not reveal any similarity between these isolates and the Klebsiella strains orally fed to the animals (Figure 2).
Klebsiella was found in the lungs of the animals provided with enteral formula contaminated with the six strains (GIII to GVI) (Figure 1). Four days after inoculation, Klebsiella was detected in the lungs of the animals that received prednisone and/or carbenicillin, while nonmedicated animals showed Klebsiella two days after inoculation (Figure 1). RAPD analysis on mucoid colonies isolated from the lungs revealed
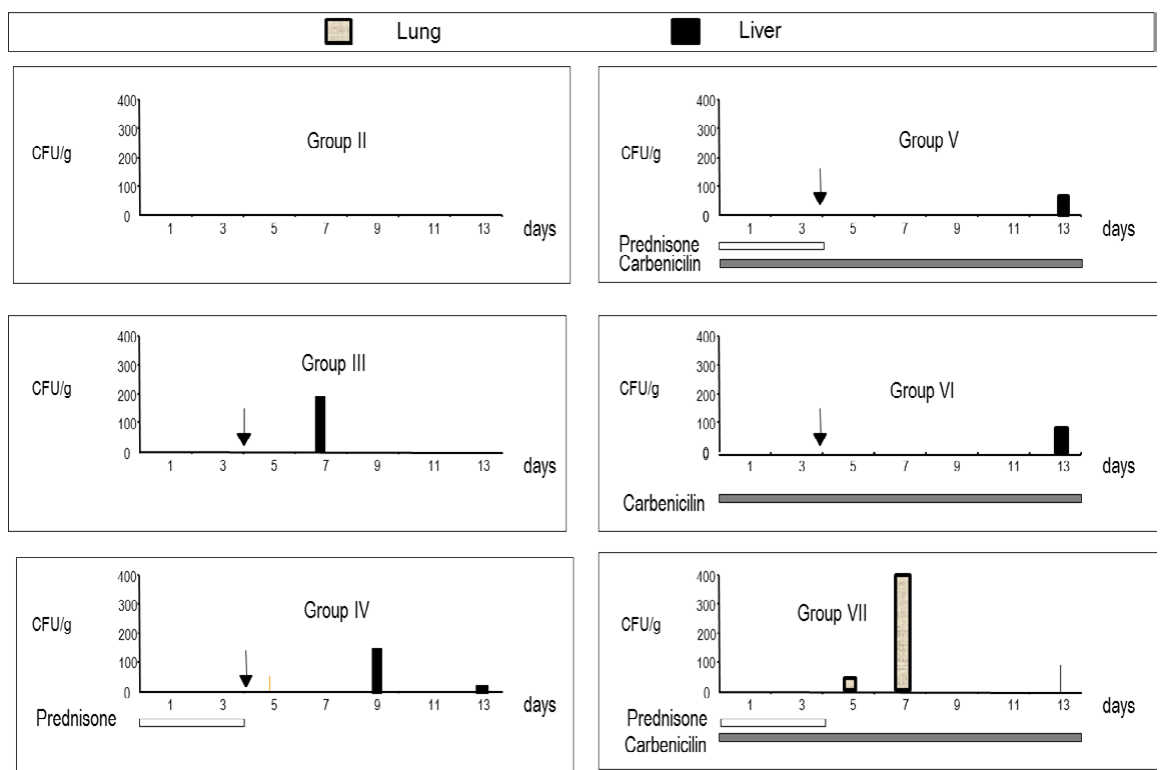

Figure 1: Klebsiella counts recovered from different organs of mice inoculated with the Klebsiella pool. The arrows represent the day of inoculation and $\square$ - Lung, $\mathbf{-}$ - Liver. See Materials and Methods for treatment description.
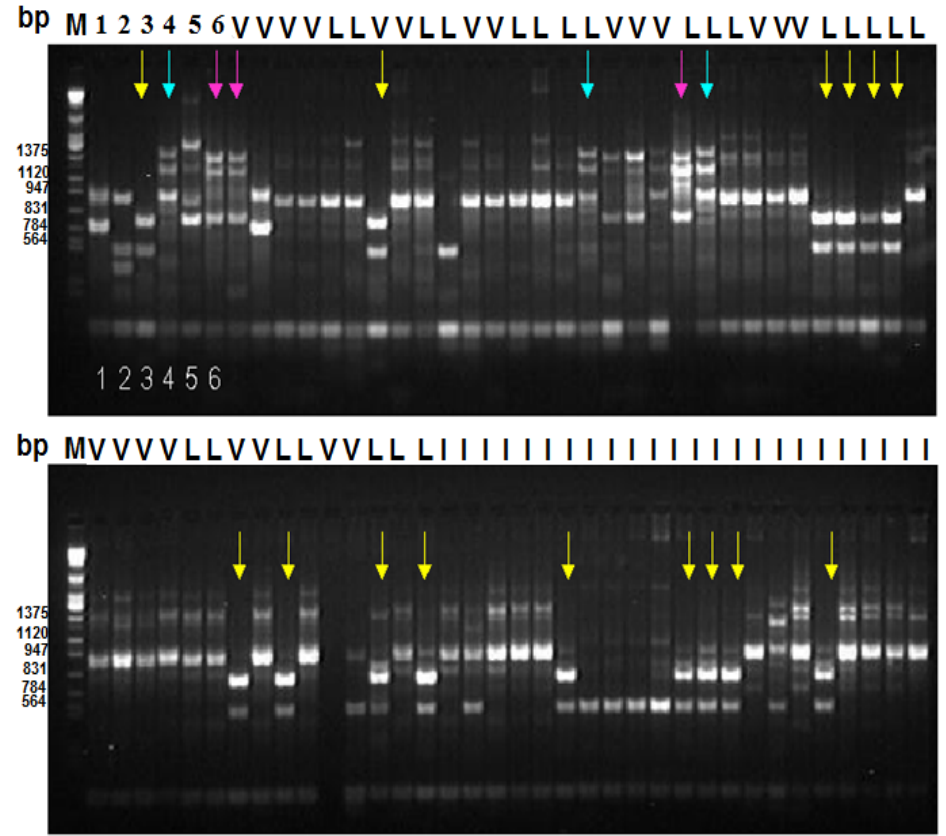

Figure 2: RAPD analysis of total DNA extracted from Klebsiella strains with the OPD20 primer. Numbers 1 to 6 refers to Klebsiella strains administered to mice. See Table 1 for strain description. Kidney, heart, lung, spleen, liver, and gut are represented by the letters $\mathrm{K}, \mathrm{H}, \mathrm{L}, \mathrm{S}$, $\mathrm{V}$, and I, respectively. Isolates with identical RAPD profile are shown by the same arrows. DNA of phage Lambda restricted with the enzymes HindIII, EcoRI, and BamHI, in base pairs (M). 
that these bacteria had the same band profile as the Klebsiella strains fed to animals in Groups III and IV (Figure 2).

Higher counts of typical Klebsiella colonies in samples collected from the intestines of animals in Groups IV to VII were noted (Table 2). These mice all received prednisone and/or carbenicillin. However, the highest colony count was obtained when both drugs were administered together with the Klebsiella pool.

\section{Discussion}

Bacterial translocation is a phenomenon that seems to explain the origin of many infections caused by Klebsiella in hosts with dysregulated immune response. In this study, we confirmed that Klebsiella translocation does not occur in hosts with a healthy immune system. This was evident from the observation that no typical Klebsiella colonies were found in samples collected from the liver and lungs of animals that had not been fed with contaminated diet and had not been medicated with immunosuppressive drugs (Groups I and II, respectively). On the other hand, the results obtained from the control group (Group VII) showed that Klebsiella from the autochthonous microbial gut became translocated upon depression of the immune system and selective decontamination induced by antibiotics.

It has been well documented that antibiotic treatments cause aberrances in the host microbiota. Although it is generally believed that such changes become normalized within weeks of cessation of antibiotics, recent evidence challenges this notion. In the context of dysbiosis (an imbalance of protective and pathogenic bacteria in the gut), antibiotics can thus be viewed as a double-edged sword. They are effective in eradicating pathogens but also nonspecifically reduce microbial diversity, thereby making it possible for opportunistic bacteria to colonize the newly hospitable niches in the gut ecosystem [16]. Such is the case of Klebsiella Various disease states have been correlated with dysbiosis. Changes to the community structure of the intestinal microbiota are not without consequences, considering the wide effects that microbes have on both local and systemic immunity. Microbiotas associated with disease are defined by lower species diversity, fewer beneficial microbes and/or the presence of pathobionts [11].

In this study, the highest number of Klebsiella colonies was detected in the liver (Groups III-VI). This may have been because the gut and liver are connected by the portal venous system, which makes the liver more vulnerable to translocation of bacteria, bacterial products, endotoxins or secreted cytokines [17]. Thus, the presence of Klebsiella in the livers of the animals analyzed differed over the sampling period, which suggested that it is difficult for infectious processes to become established. This is because the mesenteric lymph node is the first line of defense, once the intestinal barrier has been breached [18].

The presence of Klebsiella in lung samples (Group VII) from non-medicated mice may be due to disequilibria in the commensal

\begin{tabular}{|c|c|c|}
\hline Groups & $\begin{array}{c}\text { Klebsiella count } \\
\text { (CFU/g gut) }\end{array}$ & $\begin{array}{c}\text { RAPD profile similarity with } \\
\text { isolates administered to mice }\end{array}$ \\
\hline II & $3.5 \times 10^{2}$ & ND* \\
\hline III & $5.7 \times 10^{2}$ & ND \\
\hline IV & $4.5 \times 10^{3}$ & Isolate 3 (P15) \\
\hline V & $2.3 \times 10^{3}$ & ND \\
\hline VI & $6.6 \times 10^{4}$ & Isolate 3 (P15) \\
\hline VII & $3.9 \times 10^{3}$ & ND \\
\hline
\end{tabular}

${ }^{*} \mathrm{ND}$ - not detect

Table 2: Colonization of the intestinal tract by the Klebsiella pool. microbiota of the gut. Current evidence shows that dietary factors affect the microbial ecosystem in the gut. Contaminated diets and environmental changes may be responsible for this, though reducing the resistance to colonization by exogenous bacteria. This promotes bacterial growth and represents one of the major causes of infections by opportunistic bacteria [11].

In this regard, the presence of Klebsiella in enteral diets needs to be considered as a possible source of contamination for hospitalized patients. This arises because enteral diets are especially prescribed for patients who are concentrated in intensive care units, where they are highly susceptible to nosocomial infections. These are patients who are subject to the intrinsic risks of nosocomial infections relating to complex underlying diseases, nutritional vulnerability, extreme ages (premature babies and elderly people) and the effects of immunosuppressant and/ or immune depressive drugs and broad-spectrum antimicrobial agents. Furthermore, these patients are subject to extrinsic risks such as having a prolonged stay in the hospital environment, being subjected to routines of invasive procedures and lacking guaranteed quality in all hospital services, given the complex nature of hospital organizations [19].

Corroborating these findings, the study by Calbo et al [7] described the epidemiology of and control measures taken in relation to a foodborne nosocomial outbreak of K. pneumoniae in an acute-care hospital. Their report described rapid and widespread dissemination of an epidemic clone of $K$. pneumoniae, which caused a large nosocomial outbreak through the food chain. To our knowledge, this was the first report to provide insight on how transmission of K. pneumoniae can occur through food, as the vehicle in the hospital setting. The outbreak was stopped only after control measures were applied in the kitchen. The absence of new cases during the 14-month follow-up period suggests that these measures were effective.

Moreover, analysis on lung samples obtained from mice fed with prednisone (Group IV) detected exogenous bacteria. Use of corticosteroids significantly decreases the secretory IgA rate in the intestine and may be a plausible explanation for why these drugs ease bacterial translocation into extra-intestinal sites. Certain components of the microbiota are also likely to be critical for normal development of regulatory mechanisms that contribute towards mucosal homeostasis [10].

Our results show that colonization of the intestinal tract by Klebsiella strains isolated from enteral formulas may be favored when immunosuppressive and antimicrobial medications are used. Systemic and oral antibiotics cause overgrowth of bacteria in the gut through selective decontamination of some bacteria, such as antagonists, but also lead to colonization by resistant bacteria. In addition to eliminating pathogenic microorganisms, broad spectrum antibiotics also destroy the normal microbiota, thereby allowing an increase in the number of opportunistic bacteria, usually from the family Enterobacteriaceae [20]

This is because the microbiota plays a fundamental role in induction, training and functioning of the host immune system. In return, the immune system has largely evolved as a means of maintaining the symbiotic relationship between the host and these highly diverse and evolving microbes. When operating optimally, this immune system-microbiota alliance allows induction of protective responses to pathogens and maintenance of the regulatory pathways involved in continuation of tolerance to innocuous antigens. However, overuse of antibiotics, changes in diet and elimination of constitutive partners such as nematodes may have selected a microbiota that lacks 
Citation: Pereira SCL, Maria Cristina DV (2015) Translocation of Klebsiella from Enteral Diets: Study through Mouse Model System and Molecular Technique. J Food Process Technol 7: 542. doi:10.4172/2157-7110.1000542

the resilience and diversity required for establishing balanced immune responses. This phenomenon has been proposed to account for some of the dramatic rise in autoimmune and inflammatory disorders in parts of the world where the symbiotic relationship with the microbiota has been most affected [20].

Clinical studies on diseases have identified dysbiosis in patients with inflammatory bowel disease, including both Crohn's disease and ulcerative colitis. In addition to local diseases, intestinal dysbiosis has also been correlated with systemic diseases such as obesity, diabetes, atherosclerosis and nonalcoholic fatty liver disease [11].

We have provided evidence that $K$. pneumoniae becomes translocated into the lungs and liver of test mice fed with a Klebsiella pool, and these results emphasize the importance of strict control over microbial contamination during preparation and administration of these feeds.

This was made possible through using RAPD analysis in order to discriminating between autochthonous strains and those from the enteral diet. The amplified DNA fragments were specific for Klebsiella strains in liver, lung and intestine samples. In a recent study, the discriminatory power of the pulsed field gel electrophoresis (PFGE) and random amplified polymorphic DNA (RAPD) methods for typing 54 clinical isolates of Klebsiella pneumoniae were compared. All the isolates could be typed using RAPD, while $3.6 \%$ of them could not be typed by PFGE. PFGE is considered to be the gold standard for typing K. pneumonia. The repeatability of the two typing methods was $100 \%$, with satisfactory reproducibility. It was concluded that an optimized RAPD protocol is technically less demanding and time consuming, which makes it a reliable typing method that is competitive with PFGE $[21,22]$. It should be emphasized that dissemination of data analyses such as that of the present study and other recent studies should be done routinely among all the healthcare and administrative professionals of hospital institutions. Many professionals will, through becoming aware of the results relating to their own specific services, start to rethink their practices and become more involved in prevention and control practices, with surveillance in its true sense.

\section{References}

1. Thornton MM, Chung-Esaki HM, Irvin CB, Bortz DM, Solomon MJ, et al. (2012) Multicellularity and antibiotic resistance in Klebsiella pneumoniae grown under bloodstream-mimicking fluid dynamic conditions. J Infect Dis 206: 588-595.

2. Meatherall BL, Gregson D, Ross T, Pitout JD, Laupland KB, et al. (2009) Incidence, risk factors, and outcomes of Klebsiella pneumoniae bacteremia. Am J Med 122: 866-873.

3. Fung CP, Lin YT, Lin JC, Chen TL, Yeh KM, et al. (2012) Klebsiella pneumoniae in gastrointestinal tract and pyogenic liver abscess. Emerg Infect Dis 18: 13221325

4. Lin YT, Liu CJ, Fung CP, Tzeng CH (2011) Nosocomial Klebsiella pneumoniae bacteraemia in adult cancer patients - characteristics of neutropenic and nonneutropenic patients. Scand J Infect Dis 43: 603-608

5. Hurrell E, Kucerova E, Loughlin M, Caubilla-Barron J, Hilton A, et al. (2009) Neonatal enteral feeding tubes as loci for colonisation by members of the Enterobacteriaceae. BMC Infect Dis 9: 146-154.

6. Okuma T, Nakamura M, Totake H, Fukunaga $Y(2000)$ Microbial contamination of enteral feeding formulas and diarrhea. Nutrition 16: 719-722.
7. Calbo E1, Freixas N, Xercavins M, Riera M, Nicolás C et al. (2011) Foodborne nosocomial outbreak of SHV1 and CTX-M-15-producing Klebsiella pneumoniae: epidemiology and control. Clin Infect Dis 52: 743-749.

8. Fertas-Aissani R, Messai Y, Alouache S, Bakour R (2012) Virulence profiles and antibiotic susceptibility patterns of Klebsiella pneumonia strains isolated from different clinical specimens. Pathologie Biologie 61: 209-216.

9. Wiest R, Garcia-Tsao G (2005) Bacterial translocation (BT) in cirrhosis Hepatology 41: 422-433.

10. Peloquin JM, Nguyen DD (2013) The microbiota and inflammatory bowe disease: insights from animal models. Anaerobe 24: 102-106.

11. Chan YK, Estaki M, Gibson DL (2013) Clinical consequences of diet-induced dysbiosis. Ann Nutr Metab 63 Suppl 2: 28-40.

12. Rashid T, Wilson C, Ebringer A (2013) The Link between Ankylosing Spondylitis, Crohn's Disease, Klebsiella, and Starch Consumption. Clinical and Developmental Immunology.

13. Sánchez E, Donat E, Ribes-Koninckx C, Fernández-Murga ML, Sanz Y, et al (2013) Duodenal-Mucosal Bacteria Associated with Celiac Disease in Children. Applied and Environmental Microbiology 79: 5472-5479.

14. Bagley ST, Seidler RJ (1978) Primary Klebsiella identification with MacConkeyinositol-carbenicillin agar. Appl Environ Microbiol 36: 536-538.

15. Martins ML, Araújo EF, Mantovani HC, Moraes CA, Vanetti MC, et al. (2005) Detection of the apr gene in proteolytic psychrotrophic bacteria isolated from refrigerated raw milk. Int J Food Microbiol 102: 203-211.

16. Jernberg C, Löfmark S, Edlund C, Jansson JK (2007) Long-term ecological impacts of antibiotic administration on the human intestinal microbiota. ISME J 1: $56-66$

17. Quigley EM, Stanton C, Murphy EF (2013) The gut microbiota and the liver Pathophysiological and clinical implications. J Hepatol 58: 1020-1027.

18. Henao-Mejia J, Elinav E, Thaiss CA, Licona-Limon P, Flavell RA, et al. (2013) Role of the intestinal microbiome in liver disease. J Autoimmun 46: 66-73.

19. Viale P, Giannella M, Lewis R, Trecarichi EM, Petrosillo N, et al. (2013) Predictors of mortality in multidrug-resistant Klebsiella pneumoniae bloodstream infections. Expert Review of Anti-infective Therapy. 1: 1053-1063.

20. Belkaid Y, Hand TW (2014) Role of the Microbiotain Imm unity and Inflammation. Cell 27: 121-141.

21. Ashayeri-Panah M, Eftekhar F, Ghamsari MM, Parvin M, Feizabadi MM, et al. (2013) Genetic profiling of Klebsiella pneumoniae: comparison of pulsed field gel electrophoresis and random amplified polymorphic DNA. Braz J Microbio 44: 823-828.

22. Li W, Raoult D, Fournier PE (2009) Bacterial strain typing in the genomic era FEMS Microbiol Rev 33: 892-916. 